\title{
PROCEEDINGS OF THE THIRD ANNUAL MEETING OF THE CENTRAL SOCIETY FOR CLINICAL RESEARCH HELD IN CHICAGO, NOVEMBER 21, 1930
}

\author{
Focal Necrosis of the Liver. By Wm. J. Dieckmann, M.D., St. Louis, Mo.
}

Eddington in a report of focal necrosis of the liver as a result of ligation of the common duct and infection with $B$. aertrycke, noted that one of the controls with ligation of the common bile duct, happened to be well advanced in pregnancy. It died four days after the operation and although its liver showed well marked focal necrosis, careful cultural investigations were negative.

We have produced focal necrosis of the liver by feeding meat to dogs and injecting tissue fibrinogen in the peripheral circulation. Lesions produced in this way showed a peripheral hemorrhage and necrosis, the lesion characteristic of eclampsia.

Eddington's experiments have been repeated, using chiefly pregnant guinea pigs near term. Marked liver lesions have been produced, some showing hemorrhage, but the majority pure necrosis. The lesions have been mostly central and mid zonal.

Untoward Effects of Intravenous Sodium Chloride Solution in Severe Renal Insuffciency. By E. G. WAKEFIELD, M.D., and N. M. KeITH, M.D., Rochester, Minn.

Two cases of chronic glomerulonephritis with severe renal insufficiency showed an intolerance to the intravenous administration of sodium chloride. Both had a blood creatinin of $12 \mathrm{mgm}$. per cent on admission. In both, the prolonged intravenous injection of ten per cent glucose solution containing one per cent sodium chloride resulted in anuria or oliguria. Subsequent administration of glucose solution alone resulted in a satisfactory urinary excretion. These findings have both theoretical and practical significance.

Experimental Edema. By S. A. Shelburne, M.D., and W. C. Egloff, M.D., (by invitation) and M. A. Blankenhorn, M.D., Cleveland, Ohio.

Edema was produced in a dog by a diet low in protein but adequate in carbohydrate, fat, and vitamin. Edema was also produced rapidly by the selective removal of plasma protein in dogs that were fed the same diet. The chemical, pathological, and clinical findings in these dogs were almost identical and resembled starvation edema (Der Oedemkrankheit) more nearly than any other syndrome.

Further evidence of the greater importance of the sodium ion as compared to the chloride ion was added since sodium chloride and sodium bicarbonate increased edema while potassium chloride did not. 
Evidence is presented to show that the chloride excreting power of the kidney is normal in experimental edema of low plasma protein origin.

The Renal Lesions in Dogs With Experimental Hypoproteinemia. By LouIs LEITER, M.D., Chicago, Ill.

A large series of kidneys has been studied for lesions attributed by other investigators to hypoproteinemia. Various changes, including extensive fibrosis of the kidney, have been observed in the control animals as well as in those undergoing plasmapheresis. There was no correlation between the duration of the hypoproteinemia and the degree of renal pathology. The observed lesions are indistinguishable from "spontaneous" nephritis of dogs. No resemblance exists between renal lesions in dogs and those of so-called nephrosis in man. It may be concluded that the renal lesions of plasmapheresis have nothing to do with nephrotic contracted kidneys in man.

Experimental Chronic Renal Insufficiency in Dogs, with Special Reference to Arterial Hypertension. By CaRL W. Apfelbach, M.D., and Clyde Reynolds JENSEN, M.D., (by invitation), Chicago, Ill.

In recent years, there has been an increasing tendency to interpret the clinical manifestations of Bright's disease as part of a general disease of the body, in contrast to the conception formerly held by many, that the alterations in the body were solely due to the changes in the kidneys.

It was deemed worth while, therefore, to develop experimentally a form of renal insufficiency that would be dependent entirely on changes in the kidneys. To accomplish this result, a method was chosen that would injure the glomeruli of kidneys, inasmuch as the severe forms of Bright's disease of human beings are presumably initiated in the glomeruli.

Particles of charcoal, ground and sieved so as to obtain pieces large enough to occlude the glomerular tufts, were injected into the renal arteries of dogs. By this procedure, reduction in kidney tissue was induced without injuring other tissues in the body. It was concluded that the clinical alterations which resulted in the experimental animals revealed the changes that might be expected in human beings as a result of reduction in the quantity of renal tissue. It was further concluded that the application of this syndrome would indicate, in humans, the rôle played by the kidneys in producing the symptoms found in patients suffering from some of the signs of Bright's disease.

There were secured from ninety experimental animals several that lived from several weeks to over one year. A definite group of alterations were observed and these were:

Decrease in the ability of the kidneys to concentrate and dilute urine.

Nitrogen retention in the blood stream.

Polyuria.

Decrease in body weight.

Decrease in the carbon dioxide combining power of the blood. 
Edema and arterial hypertension did not occur.

Severe anemia, like that observed in subacute and chronic glomerular nephritis, was not observed.

It is concluded, that death cannot be ascribed to renal insufficiency, unless this syndrome occurs.

Further Observations on Climatic Effects, with Particular Reference to Seasonal Alterations in the Human Conception Curve. By C. A. Mrlls, M.D., Cincinnati, Ohio.

There will be given a brief review of the previous findings regarding the relationship of climate to such diseases as diabetes mellitus, pernicious anemia, exophthalmic goiter, Addison's disease and angina pectoris. The low mortality from these diseases in the tropics, sub-tropics and orient will be stressed, as well as their high, and increasing mortality rate in the cooler temperate regions.

Since these findings seemed to point to the endocrine system as being most susceptible to climatic influences, it was deemed advisable to see if the sex glands exhibited like effects of climatic stimulation or depression. Monthly birth statistics were collected from various units of population, the months equalized to a 31day basis, and the births of each month transposed back to the time of conception. The graphs to be shown are thus the "conceptions resulting in live births," with the mean of each month for several years plotted to show the yearly seasonal variations.

The data thus presented leaves no doubt that at least one important climatic factor, temperature, exerts a marked effect on human fertility. The conception rate is highest when the monthly mean temperature is around $60^{\circ} \mathrm{F}$, while above $70^{\circ} \mathrm{F}$. and below $50^{\circ} \mathrm{F}$. there occurs a progressive depression in fertility. Evidence is not yet at hand to determine which sex is responsible for these effects, or whether both are equally so. Proper control data are presented to rule out certain other possible factors, such as frequency of intercourse, variations in the marriage rate, etc.

Further Observations on the Effect of Viosterol on Platelets. By LAWRENCE D.

Thompon, M.D., and Lillian Hadseli. (by invitation), St. Louis, Mo.

The increase of platelets following exposure to sunlight has been noted for some time. Phillips and Robertson demonstrated that oral administration of viosterol causes a rise in platelet count in the rat. Recently, announcement was made that the administration of viosterol to normal individuals was followed by a similar rise in platelet count. It was also stated that the platelet count could be markedly increased in various hemorrhagic diseases and that the increase was accompanied by decrease in bleeding and coagulation time but without alteration in the symptomatology or course of the disease. The study has been continued, the series of cases has been augmented. In addition, two other blood dyscrasias have been included. 
A New Method of Interpreting Acid-Base Data. By A. BaIRd Hastings, M.D., Chicago, Ill.

By the use of triaxial coördinates an acid-base chart has been devised which permits the evaluation of the acid-base condition of an individual when the $\mathrm{pH}$ and $\mathrm{CO}_{2}$ of the blood is known. Such a chart separates disturbances of the acidbase balance which have their origin in a disturbance of respiratory function from those which have their origin in disturbances of excretory function.

The distance which a point on such a chart lies from the normal point of reference and the angle which the line connecting these two points makes with the line of reference can be interpreted as indicating the magnitude of and the qualitative nature of the observed disturbance in the acid-base balance.

\section{Chemical Changes in Blood Plasma and Cells in Intestinal Obstruction, with Special} Reference to the Distribution of Phosphorus. By George Martin Guest, M.D., and Wrlliam De Wrtt Andrus, M.D., Cincinnati, Ohio.

The changes which occur in the blood plasma during high intestinal obstruction have been well defined, the loss of $\mathrm{Cl}$, and lesser loss of $\mathrm{Na}$, with increase of $\mathrm{HCO}_{3}$, being the most noteworthy of the changes. Little attention has been paid to the cells, however, and of the blood $\mathrm{P}$ only the inorganic fraction has been considered in relation to changes in the acid-base equilibrium.

In this study the distribution of blood $\mathrm{P}$ was measured as the (1) inorganic, (2) organic acid-soluble, (3) lipin, and (4) total $\mathrm{P}$ of oxalated whole blood and plasma. Parallel measurements were made of the erythrocyte count, relative cell volume, hemoglobin, total protein, NPN, sugar total base, $\mathrm{Na}, \mathrm{K}, \mathrm{Cl}$, and $\mathrm{CO}_{2}$. By means of the relative cell volume, values for the intracellular chemical constituents were calculated and changes in the cell electrolytes were demonstrated which are comparable but not always equivalent to those noted in the plasma electrolytes.

In experimental high intestinal obstruction in dogs the total $P$ was increased in both cells and plasma. In a typical experiment the total $\mathbf{P}$ of the cells increased from $72 \mathrm{mgm}$. per cent to $93 \mathrm{mgm}$. per cent. This increase was mainly of the acidsoluble organic $P$ within the cells and, to a less extent, the inorganic $P$ of both cells and plasma. It seems likely that this organic $\mathbf{P}$ fraction may be actively concerned in the acid-base equilibrium of the cells.

Bacteriologic Studies in Thrombo-angiitis Obliterans. By B. T. HorTon, M.D., and A. H. E. Dorsey, M.S., (by invitation), Rochester, Minn.

The work which we are reporting was started in November, 1927, and has continued for a period of two and one-half years. Acutely inflamed veins and arteries were obtained from biopsies or amputations in thirty-four cases of thrombo-angiitis obliterans. Gram-positive pleomorphic streptococci were obtained in pure culture from nine cases and green producing streptococci from two cases. Negative cultures were obtained from arteries and veins from twenty-four normal subjects. Various methods of inoculating these organisms into 165 rabbits and three dogs 
have been used, and pathologic lesions have been produced in a small number of rabbits, which from the gross and microscopic appearance are identical to those which we accept as that of thrombo-angiitis obliterans. This study, though incomplete, suggests that thrombo-angiitis obliterans is an infectious disease, and that these organisms may be of etiologic significance.

Some Clinical Observations on the Use of Theobromine in Peripheral Vascular Disease. By George W. ScuphaM, M.D., Chicago, Ill.

The success attending the use of purin base derivatives in angina pectoris suggested its trial in peripheral vascular disease.

Criteria for observation were subjective response, appearance, and measurement of the temperature of the affected extremities under standard conditions by means of a thermoelectric couple.

Observations shown are on typical cases of Buerger's disease, arteriosclerosis with intermittent claudication or tissue impairment, Raynaud's disease, acrocyanosis, hypertension and normals.

A case of arteriosclerosis with local ischemia observed for more than a year showed definite objective and subjective improvement during periods of medication with a fall in the temperature curves when theobromine was discontinued. In Buerger's disease good results were obtained in the earlier stages; none in the advanced case. There was no demonstrable improvement in Raynaud's, or in acrocyanosis. Normals showed little effect.

Results seemed to show that theobromine was effective in organic disease of the larger arteries when the symptoms and nutritional changes are best explained on the basis of angiospasm.

Lead Encephalitis. By Robert A. Kehoe, M.D., (by invitation) and C. A. Mriss, M.D., Cincinnati, Ohio.

Experimental and clinical evidence is presented to indicate that the cerebral manifestations of lead poisoning are due to the localization of lead in the brain tissue, rather than in the meninges, or in the blood circulating through the brain.

It is further shown that lead forms a stable and insoluble compound in the brain under the customary conditions of physiological activity. This suggests that such lead as is brought to the brain through the circulation is quantitatively bound.

Certain tentative conclusions as to the conditions under which saturnine encephalopathy develops, and certain suggestions as to the treatment of lead poisoning in such a manner as to spare the nervous tissues, arise from these considerations.

The Relationship Between Oxygen Consumption and Nitrogen Metabolism in Pernicious Anemia and Leukemia. By C. W. BALDRIDGe, M.D., and A. P. BARER, Ph.D., (by invitation), Iowa City, Iowa.

In pernicious anemia and in leukemia a definite relationship between oxygen consumption and nitrogen metabolism has been demonstrated. During periods of rapid cell destruction, especially if there be a negative nitrogen balance (relapse in 
pernicious anemia or soon after roentgen ray treatment in leukemia) the oxygen consumption is increased. The consumption of oxygen decreases while nitrogen is being stored in spite of the fact that young cells are being formed rapidly (e.g., during remissions in pernicious anemia or several days after roentgen ray treatment in leukemia). The total oxygen consumption would probably be more increased after roentgen ray treatment were it not for the loss in the urine of incompletely metabolized protein.

Observations on Some Conditions Causing $R-T$ Deviations Characteristic of Coronary Occlusion. By Louis N. Katz, M.D., Chicago, and A. W. Wallace, M.D., (by invitation), Cleveland, Ohio.

Recent observations by Scott, Katz and Feil have shown that pericardial effusions will lead to R-T deviations characteristic of coronary occlusion. Animal experiments have shown that ligation of a coronary artery will not lead to such R-T changes until cardiac incompetence sets in. (Feil, Katz, Moore and Scott.) Three cases are presented in this report to illustrate the rôle cardiac incompetence plays in producing the characteristic R-T deformities. One case shows the effect of cardiac incompetence superimposed on an acute coronary thrombosis. The second shows the effect of cardiac incompetence in the presence of coronary sclerosis but with no thrombosis present. The third shows the effect of acute myocardial incompetence added to a slight pericardial effusion. In all three cases typical R-T deviations are present. In each the cardiac incompetence was the primary and major cause for these electrocardiographic changes.

Interpretation of the Initial Deflections of the Ventricular Complexes of the Electrocardiogram. By A. G. Macleod, M.D., PAUL S. BARker, M.D., and F. N. WILson, M.D., Ann Arbor, Mich.

Obviously, the electrocardiogram recorded in the three standard leads must be the resultant of many opposed, or partially opposed, potential differences which occur in the heart during the inscription of the QRS complex. It must, therefore, be that only those forces which are unopposed, or only partially opposed, can produce measurable potential differences at the surface of the body. By applying these theoretical conceptions to a detailed anatomical study of the canine and human heart it is possible to show that the results of Barker, Macleod and Alexander, who believe that what is, at present, called right bundle branch block, is in reality left, and vice versa, are the ones to be expected. It is also possible to reinterpret the observations of Lewis so as to show that the above authors' views are in conflict only with Lewis' interpretation of his data, and not with the data itself. Rational explanations of bundle branch block curves and preponderance curves can be made on this basis.

The Treatment of Patients with Addison's Disease with the Extract of the Suprarenal Cortex Prepared by Swingle and Pfiffner. By Leonard G. Rowntree, M.D., and CARL H. Greene, M.D., Rochester, Minn. 
An extensive experience in the use of the so-called Muirhead regimen in the treatment of patients with Addison's disease has convinced us of the futility of ordinary therapeutic measures in combating the crises of acute adrenal insufficiency, and also of the great need for a more active cortical preparation which could be administered either hypodermically or subcutaneously. In March, 1930, Swingle and Pfiffner announced the preparation of an aqueous extract of the suprarenal cortex which would maintain the life of bilaterally adrenalectomized cats indefinitely, and later they reported that by the administration of this extract they were able, not only to revive comatose animals, prostrate and on the verge of death from adrenal insufficiency, but also to restore them to a normal condition and to keep them in perfect health. The importance of such an announcement and the interest aroused by the possibility of using this extract in clinical medicine is obvious.

The preparation which we are using now is almost free from epinephrine, is suitable for intravenous administration and is almost nonirritating locally. We have used this in the treatment of six patients with Addison's disease. The results in these cases convince us of the efficacy of this cortical hormone. The disappearance of anorexia, increase of appetite, the gain in weight and the definite euphoria were striking in all cases. As long as the preparation could be administered, the results were all that could be desired. Our supply of the preparation has been extremely meager and intermittent, so that we have not been able to observe the results of consistent dosage and continued administration, but the immediate results in a crisis are excellent. The disease, however, is chronic, and it will be necessary for several years to elapse before a final appraisal can be made of the value of this cortical hormone in Addison's disease.

The Intravenous Administration of Barium Chloride in Humans. By J. H. FoulGER, M.D., (by invitation), and JoHnson MCGuIRE, M.D., Cincinnati, Ohio.

Considerable interest has been aroused by the work of Cohn and Levine on the use of barium chloride in cases of heart block with Stokes-Adams attacks. Several papers on the subject have appeared subsequently, but the results have been variable and the drug has hitherto been given exclusively per os. The slowness of its absorption and perhaps in some cases the almost complete or complete lack of absorption might account for its failure. The desirability of securing therapeutic effects promptly in Stokes-Adams syndrome is apparent. Therefore, it seemed of interest to study the effect of intravenous administration of the drug, using, of course, a much lower dosage than that advised orally.

The characteristic effect on the human and animal heart were studied electrocardiographically, and these findings are described.

A Study of Five-Hundred Diabetic Patients. By Elmer L. Sevringhaus, M.D., Madison, Wis.

These patients, seen in the eight years since insulin has been available, have had insulin only when necessary to control the glycosuria, (or to maintain blood sugar 
levels more nearly normal in a few younger individuals). Diets have been planned according to Woodyatt's rule, $\mathrm{F}=2 \mathrm{C}+0.5 \mathrm{P}$. The maintenance standard has been at the accepted normal weight for the patient, and with no limitation on occupation. Under such conditions 57 per cent required insulin at discharge from the hospital.

Ketosis was observed in the first urine examined in the hospital in 58 per cent of the patients. Insulin was required at discharge by $\frac{5}{6}$ of this group. Of the 42 per cent without ketosis, only $\frac{1}{b}$ required insulin. In no case of this latter group was the insulin large. Therefore, the occurrence of ketosis with glycosuria at the time of the first examination may be accepted as a prognostic indication that insulin will be needed, although later improvement may render its continuance unnecessary. This information saves time in planning treatment.

Of the 500 cases 90 are known to have died. Causes, which are determined in 75 of these, include, in order of importance: sepsis, cardiovascular-renal disease, coma, and carcinoma. Septic processes include, in the following order: pneumonia, gangrene, tuberculosis, gas bacillus infection after amputation, and a miscellaneous group. In ten of the cases neglect on the part of the patient or parent after instruction and discharge from the hospital is directly responsible for death. No uncomplicated case of coma has been lost in the hospital.

In the entire series, routine histories reveal reduced or absent patellar reflexes in 45 per cent. Pains in the extremities (neuritis?) are recorded in 34 per cent. These pains include cases in which vascular conditions are probably primarily at fault. There are 37 per cent of these cases with visual disturbances, excluding cataracts and refraction errors acquired before diabetes. This visual error is often relieved by the diabetic therapy itself. In any case, lens fitting should not be undertaken within less than one month after the patient is sugar free.

\section{A Study of Blood Phosphate and Blood Glucose Curves After the Oral Administration} of 100 Grams of Glucose. By Henry J. John, M.D., and D. Roy McCulLAGH, Ph.D., (by invitation), Cleveland, Ohio.

The curve of phosphate and glucose after the administration of 100 grams of glucose orally to sixty patients has been studied. The statement that they are related is confirmed, but we find that the so-called typical diabetic $\mathrm{PO}_{4}$ curve is not always in itself diagnostically reliable. If we depend upon phosphate curves alone for diagnosis an error of 19 to 67 per cent is incurred, depending upon the condition of the group under consideration. In hyperthyroidism the errors are from 40 to 67 per cent.

The excretion of phosphates bears no relation to the urine output so that we can consider that the phosphate excretion is not a pure mechanical washing out of phosphate, depending on the increase of urine excretion, but a function of a metabolic process, totally independent of the amount of urine excreted. In diabetics the phosphate excretion is markedly increased. 
Diabetes Mellitus Associated with Addison's Disease. (Report of Two Cases.) By

Frank N. Allan, M.D., Rochester, Minn.

There has been much speculation regarding the relationship of various endocrine glands to diabetes, and the rôle of the adrenals has received especial consideration. The theory that diabetes might be due to excessive activity of the adrenals was formerly strongly supported. Recent investigations showing that continuous administration of epinephrine may cause suppression of oxidation of glucose have revived this idea.

Two cases of diabetes associated with Addison's disease are reported. This combination represents a rare clinical picture. It also suggests various problems of theoretical interest.

Jerusalem Artichokes and Liver in the Treatment of Diabetes Mellitus. By SAMUEL Sosknn, M.D., and H. F. BrNSwanger, M.D., (by invitation), and Solomon STrouse, M.D., Chicago, Ill.

The experimental use of Jerusalem artichokes and of liver in the treatment of diabetes mellitus is reported. No evidence was obtained that these substances are of any therapeutic value in this condition. Each patient was hospitalized throughout the experiment, and judgment was chiefly based on daily urinary sugar and nitrogen excretion over adequate periods rather than on temporary changes in blood chemical values or alterations in respiratory exchange. Adequate control periods on orthodox diets, with or without insulin, were instituted in all cases, the artichokes or liver being then substituted in the diet so as to leave the caloric intake and the distribution of calories between protein, fat and carbohydrate unchanged. The results of other workers are discussed in the light of the work here presented.

Quantitative Relationships Between Dosage and Therapeutic Response in 100 Patients with Pernicious Anemia Treated with Liver Extract of Desiccated Stomach. By Cyrus C. Sturgis, M.D., and Raphael IsaAcs, M.D., Ann Arbor, Mich.

The rate of response to therapy was observed in 50 patients with pernicious anemia treated with liver extract and $\mathbf{5 0}$ similar patients treated with desiccated stomach. The average maximum reticulocyte percentage reached in the former group was 22.36 per cent (calculated 23.43 per cent) and in the latter group 22.63 per cent (calculated 22.58 per cent). The maximum reticulocyte percentage was reached in 6.9 days by the liver treated group and in 7.84 days by the stomach treated patients. This is attributed to the slower absorption of the non-soluble desiccated stomach. A suboptimal dose, infection, gastric retention, or blood transfusion decreases the observed maximum reticulocyte percentage below the calculated height. A massive dose decreases the latent period and increases the height of the reticulocyte rise. Within limits, the larger the dose, the shorter the time required for the red blood cell count to reach normal.

Chronic Ulcerative Colitis. (Further Studies.) By M. H. Streicher, M.D., (by invitation), and C. S. Wriliamson, M.D., Chicago, Ill. 
Until recently, all reports on the subject of ulcerative colitis were discouraging. For many years it has been the opinion of most gastroenterologists that the treatment of colitis was mainly that of the specific forms of ulcerative colitis and that the diagnosis of the nonspecific ulcerative colitis was based on the existence of colon ulcers with negative bacteriologic observations.

Method of procedure. In the past three years we have carried on a careful survey of all cases of diarrhea observed in our gastro-intestinal clinic, first, to confirm or refute the observations of Bargen and, secondly, to determine the specificity and therapeutic value of autogenous vaccine. The patients were properly prepared a day before and routine proctoscopic examinations were made in each patient. Cultures were then obtained through the proctoscope by means of a sterile swab and transferred at once to a proper medium for incubation and subsequent culture and subculture. The individual strains of bacteria were then isolated from the cultures and a polyvalent vaccine was prepared. The autogenous vaccine was then administered hypodermically to the patients in progressively increasing dosages in accordance with the severity of the infection.

Results. Briefly summarized, the results clearly demonstrate that most remarkable improvement was noted in about 80 per cent of the cases observed.

Our results show that in most cases of ulcerative colitis green-producing grampositive diplococci are found predominating. Frequently these are in association with other bacterial flora.

In addition to the green-producing gram-positive diplococci and hemolytic streptococci, we found other bacteria in ulcerative colitis such as diphtheroid bacilli, Staphylococcus albus and aureus, Staphylococcus albus-hemolyticus, Bacillus pyocyaneus, B. lactis-aerogenes and B. coli.

Treatment. The method of approach in general was three-fold; namely, prophylactic, dietary and specific. In reference to diet, one is impressed considerably with the encouraging results obtained with an essentially high calory, high vitamin and low residue diet. This specific treatment consists of injections of polyvalent autogenous vaccine hypodermically.

Conclusions. (a) The green-producing gram-positive diplostreptococcus is not the sole etiologic factor of chronic ulcerative colitis.

(b) A polyvalent autogenous vaccine given hypodermically is of considerable therapeutic value.

(c) The green-producing gram-positive diplococci are all inulin negative and the majority are mannite negative.

Response of Secondary Anemia to Fetal Liver. By Chardes H. Watkins, M.D., and HeRbert Z. Giffin, M.D., Rochester, Minn.

In an article on the treatment of secondary anemia by Herbert Z. Giffin and Charles H. Watkins read before the Section on Pharmacology and Therapeutics at the Annual Session of the American Medical Association, Detroit, June 26, 1930, a review of the clinical results following the administration of desiccated fetal liver $n$ the treatment of 120 cases of chronic secondary anemia was presented. The 
paper submitted for presentation before the Central Society for Clinical Research is an elaboration of these data and an evaluation of the results in various types of secondary anemia as classified both from the clinical and morphologic standpoint.

Scaleniotomy in the Surgical Treatment of Pulmonary Tuberculosis. By JOSEPH W. Gale, M.D., (by invitation) and WM. S. MmDleton, M.D., Madison, Wis.

Surgical measures for the treatment of pulmonary tuberculosis involve two fundamental principles, namely physiologic rest and compression of the involved lung. The first of these indications has been met in phrenicotomy and intercostal neurectomy, which bring about paralysis of two of the most important groups of respiratory muscles. The scalene group alone of the important muscles of respiration has escaped surgical attention.

The sound clinical and experimental observations of Hoover established the respiratory function of the scaleni. By fixing the first rib they permit of an advantageous action of the upper three intercostal groups on their attached ribs with cephalad movement of the same. Paralysis of the scaleni is attended by a caudad movement of the upper three ribs on inspiratory activation of the intercostal muscles.

Because of the intercostal overactivity attendant upon phrenic paralysis the concurrent division of the scaleni was suggested to induce apical rest. The anatomic relationships render the combined operation of phrenic block and scaleniotomy quite easy. The operation has been completed in a small series of cases, both alone and in combination with phrenic block, without increased reaction or disability and with gratifying results. The indications are discussed.

\section{Analysis of One Hundred and Five Electrocardiograms Showing Changes Associated with Coronary Thrombosis. By Walter S. PRIEST, M.D., (by invitation), and W. W. HAMBURGer, M.D., Chicago, Ill.}

Those changes commonly considered indicative of coronary thrombosis are tabulated according to the lead or combination of leads in which they occur. They are inversion of $T$; positive S-T interval; high take-off of $T$; deep $Q$; distortion of the isoelectric line. In addition, slurring and notching of QRS, occurrence of positive $S-T$ without inversion of $T$, axis deviation, changes in axis deviation during a series of records, low amplitude of QRS deflection, and abnormalities of the $\mathbf{P}$ wave noted. The relation of these changes to the clinical findings is then analyzed. For this purpose the patients are grouped clinically into five classes:

(1) Those with undoubted signs of coronary thrombosis, (2) those with anginal attacks but without classical signs of thrombosis, (3) those without history of pain but with other cardiac symptoms, (4) those without pain or other cardiac symptoms, and (5) those without cardiac symptoms but with upper abdominal lesions. Patients with upper abdominal lesions and cardiac symptoms, and patients with syphilis are considered under groups 1 to 4 as indicated. Autopsy-findings are included when available.

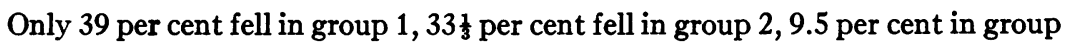


3,16 per cent in group 4, and 1.9 per cent in group 5 . Approximately 10 per cent of those in group 1 had associated upper abdominal lesions. 5.7 per cent of those in group 2 had associated upper abdominal lesions. One patient in group 2 and one in group 4 had syphilis.

\section{The Effect of Lugol's Solution on the Elevated Basal Metabolism in Conditions Other}

than Exophthalmic Goiter. By HARRY B. FrIEDGOOD, M.D., (by invitation).

(Introduced by Cyrus C. Sturgis, M.D.), Ann Arbor, Mich.

The effect of Lugol's solution in exophthalmic goiter suggested a similar investigation in other diseases characterized by an elevated oxygen consumption, notably, leukemia, polycythemia vera and acromegaly.

Symptomatic improvement occurred in all of the eight patients with chronic lymphatic leukemia and three of four patients with chronic myeloid leukemia. Aleukemic leukemia (3) and the acute leukemias (2) did not respond similarly.

Basal metabolism and pulse rates were significantly decreased in three fourths of the patients (ambulatory) with chronic lymphatic leukemia, and in one of four patients with chronic myeloid leukemia. Polycythemia vera (1) and acromegaly (1) also exhibited favorable decreases. Initial basal rates recurred under continued treatment as in exophthalmic goiter, but sometimes the depressed pulse rate lagged behind the increasing metabolism. Acute exacerbations, severe anemia and previous roentgen therapy inhibited the usual decreases. Following treatment, the leukocytes in chronic lymphatic leukemia appeared temporarily depressed, but no relation existed between rate of metabolism and leukocyte counts. Eye signs of exophthalmic goiter occurred in some of the patients with chronic leukemia.

The similarity of response to iodine suggests a non-thyrogenous disturbance, responsible for the elevated basal metabolism, and common to exophthalmic goiter, chronic lymphatic and myeloid leukemia, acromegaly and polycythemia vera.

Studies in the Circulation: Injection Method: Preliminary Report on Clinical Cases.

By J. Murray Kinsman, M.D., John Walker Moore, M.D., and W. F.

HAMLLTON, M.D., (by invitation), Louisville, Ky.

Our method for determining the output of the heart, circulation time and the amount of blood in the heart and lungs and the total blood volume has been applied to certain cases of hyperthyroidism, pernicious anemia and heart disease, both during and after recovery from the acute manifestations.

Observations on the Clinical Couirse and Effects of Treatment in 112 Cases of Portal Cirrhosis with Ascites. By C. B. Chapman, M.D., (by invitation), L. G. Rowntree, M.D., and A. M. SNell, M.D., Rochester, Minn.

The serious prognostic significance of the development of ascites in patients with portal cirrhosis is shown by the fact that $\mathbf{5 2}$ per cent of this group of patients were dead within sixteen months after the ascites appeared. It would seem that the appearance of ascites may be regarded as evidence of failing compensation in the 
portal circulation and that ascites has somewhat the same significance in portal cirrhosis that it has in cardiac disease. Treatment with diuretics is ordinarily effective in the control of ascites early in the course of the disease, but it becomes less so as time progresses. There is no type of treatment which seems to be effective in the control of the hepatic lesion itself.

Non-Specific Protein Therapy in Duodenal Ulcer. By Leon SchrF, M.D., and R. J. Norris, M.D., (by invitation), Cincinnati, Ohio.

The effect of intramuscular injections of Aolan (purified milk protein) in 27 cases of duodenal ulcer are reported.

Of these cases, 20 were ambulatory and 7 hospitalized. Those hospitalized were with one exception (case with hematemesis) not confined to bed and given a general diet. No medication was prescribed.

Marked improvement or practically complete symptomatic relief occurred in 18 or 66.7 per cent, and less marked though definite improvement in 5 or 18.5 per cent, making a total of 85.2 per cent deriving benefit.

Improvement was relatively more frequent in the colored than in the white individuals.

Improvement seemed to occur more frequently in those with normal acidity than in those with hyperchlorhydria.

A second course of injections in some instances of relapse was equally as effective as the first.

Striking gain in weight was at times noted.

Improvement seemed to occur less frequently in the presence of lues.

No change in the degree of acidity or volume of gastric juice was noted, using the method of Bloomfield and Keefer.

Patients with symptoms of less than a year's duration obtained complete relief.

The Influence of Ethyl Alcohol Upon the Gastric Absorption of Phenol in Rabbits. By F. Lowell Dunn, M.D., and Anne M. Perley, (by invitation), Omaha, Neb.

The value of alcohol lavage in phenol poisoning has been repeatedly questioned. The development of blood phenol methods permits a study of the rate of absorption of phenol with and without the addition of alcohol. These studies were made on rabbits. The free blood phenol showed a somewhat more rapid rise in a group of rabbits receiving phenol and alcohol than in a group receiving phenol alone, but the final levels were essentially the same. The conjugated phenols showed only a very slight rise.

\section{Electrocardiographic Changes Produced by Experimental Occlusion of Coronary} Vessels in the Dog. By A. R. BARNEs, M.D., Rochester, Minn.

Experiments re-investigating the electrocardiographic changes resulting from occlusion produced by ligation of various branches of the coronary arteries in dogs have been carried out. Contrary to the previous investigations it appears that 
ligation of a branch of the right coronary artery producing infarction in the right ventricle produces electrocardiographic changes which are characteristically different from those obtained when a branch of the left coronary artery is ligated.

Infarction produced in the posterior basal portion of the left ventricle in dogs does not produce electrocardiographic changes similar to those obtained following infarction in a like region in the human heart. Except for that difference it seems possible to correlate the results to date with electrocardiographic changes in man following acute coronary occlusion as described by Barnes and Whitten. It should be noted that the operative procedure and incision of the pericardium involving a possible change in the axis of the heart must be taken into account in interpreting these results and this factor has not been completely evaluated as yet.

Parasystole-A Study in the Origin and Conduction of Ectopic Impulses of Supraventricular Origin. By Julius Jensen, M.D., (by invitation), and DREw Luten, M.D., St. Louis, Mo.

Cases of so-called nodal rhythm which show premature ventricular beats following $\mathrm{P}$-waves are rare. We have studied six cases in detail. Evidence is presented that these premature beats originate in ectopic foci and interfere with the dominant "nodal" rhythm. These ventricular responses to more than one focus constitute parasystole. The conditions of parasystole with supraventricular pacemakers are studied: consideration being given to rhythmicity, rate and location of the foci; also to the block protecting the upper pacemaker and to the conditions under which this pacemaker acts on the ventricle. The probability of more than one "nodal" pacemaker in certain instances is discussed.

The hypothesis of reciprocal beating is considered, but evidence against such an explanation of our cases is presented. Most of the cases in the literature described as reciprocal rhythm fulfill the requirements of parasystole and the conception of reciprocal rhythm in any case must still be held subjudice.

Carcinomatous Bone Metastases Without Roentgen Evidence. (Special Reference to the Spinal Column). By E. L. TuoHy, M.D., Duluth, Minn.

There is a general assumption and much developing proof that the more common carcinomas metastasize at an early period to certain important bony sites. The clinical search for evidence of this disastrous bony invasion has called into use an earnest effort to show the extent and degree of such invasion through roentgen films. In prosecuting this search it has been clearly found that the roentgen changes are those produced by so-called osteoblastic changes through extra calcium deposition, and the converse, or osteoclastic changes incidental to bony replacement by soft tissue. That the prostate cancer has the faculty of setting up osteoblastic changes, and hence the creation of exaggerated calcium deposits within the bone, no one familiar with the process will longer doubt.

Hypernephroma (more recently known as carcinoma of the kidney) has the subtle faculty of invading the bones, and to a very notable degree those of the head. Carcinoma of the thyroid, as also carcinoma of the bronchus, not uncom- 
monly invades the cranial cavity as well as the cranial bones; practically all cancers, including the stomach and uterus, may metastasize into the vertebrae. Hence, the vertebral column is recognized as a crucial zone in terms of metastases, prognosis and operability.

For the purpose of this report the actual spinal column and corresponding films are shown. In three cases, though the vertebrae were badly seeded with metastases (from prostate, stomach and cervix, respectively), the roentgen evidence is negative. The sections will show that when the films were taken the process was far advanced.

Conclusion: Negative evidence of bony metastases (especially in spine) does not rule out marked invasion. In the problem of cancer control some much more subtle evidence of local and systemic invasion must be sought than any method of visualization now possible.

Surgical Operations and Associated Infections as Possible Etiological Factors in the Development of Exophthalmic Goiter and Hyperthyroidism from Adenomatous Goiter. By William A. Plummer, M.D., and Charles Mayo, 2ND, M.D., (by invitation), Rochester, Minn.

From records of The Mayo Clinic we collected thirty-eight consecutive cases in which surgical operation on some part of the body other than the thyroid gland preceded partial thyroidectomy for exophthalmic goiter or for hyperthyroidism from adenomatous goiter. Both operations were done in The Mayo Clinic. We were not particularly interested in the fact that the patients had been subjected to an operation previous to thyroidectomy except for the fact that the previous operation had caused a record of their condition to be made and that this record could be compared with that of a later time when they had demonstrated hyperthyroidism. We wished to learn if the records of the patients made during their first stay in hospital contained evidence to indicate that acute or chronic infectious processes or other factors associated with the first operation might have been etiologically related to the development of exophthalmic goiter or hyperthyroidism from adenomatous goiter. In those cases in which there was relationship in time between the development of the toxic symptoms of goiter and the first operation, the element of mere coincidence can not be excluded. Our investigation, however, tends to support the contention that infection may be an aggravating or precipitating factor in the development of exophthalmic goiter or hyperthyroidism from adenomatous goiter, in certain cases.

Basal Metabolism and Specific Dynamic Action of Foodstuffs in Normal Pregnancy. By F. Lowell Dunn, M.D., and Helen Wyandt, (by invitation), Omaha, Neb.

The respiratory gas exchange and urinary nitrogen was determined at two weeks to monthly intervals during the course of two normal pregnancies, and the puerperium. The basal metabolism data conform to the case of Sandiford and Wheeler. The specific dynamic action for carbohydrate, protein, and fat was 
determined at the same intervals. Essentially normal values were obtained throughout although the dynamic action of fat shows a steady diminution in the two cases.

Nutritional Edema. By Alexis F. Hartmann, M.D., and Milton J. Senn, M.D., (by invitation), St. Louis, Mo.

The composition of the plasma in cases of nutritional edema has been studied from the standpoint of (1) total and fractional protein concentration, (2) colloidal osmotic pressure, and (3) total acid-base equilibrium. A comparison is made with other types of edema as regards such findings, and the response to transfusions and high protein diet.

Sporadic Septic Sore Throat Due to Streptococcus Epidemicus. By I. Pıгot, M.D., Chicago, Ill.

Epidemicus septic sore throat is a well established clinical entity due to the Streptococcus epidemicus (Davis), and is spread through milk from the udder of a cow infected by this organism. In the isolation of this streptococcus certain characteristics can be brought out by the use of blood agar enriched by ascites fluid, which differentiates the streptococcus epidemicus from other hemolytic streptococci. The use of this medium has led to the ready recognition of carriers and of sporadic cases of septic sore throat. Several such patients are reported, and in addition complications such as otitis media, mastoiditis, meningitis adenitis, empyema, septic arthritis are found in other patients to be due to this streptococcus, which was often also demonstrated in throat cultures. These streptococci produce exotoxin which in skin tests with filtrates and neutralizing serums differs from the toxin of Streptococcus scarlatinae.

The Danger of Overdosage with Parathormone. By WALter M. BoothBy, M.D., Rochester, Minn.

Greenwald and Gross, in 1925, showed that the excretion of calcium in the urine was markedly increased following the administration of large doses of parathormone and sounded a warning as to the possible danger of the use of too large doses in parathyroid insufficiency.

Since then the clinical use of parathormone has become quite extensive and the doses that have been reported vary between 20 and 160 units daily.

We have studied one case in which the use of 150 units for four days was demonstrably harmful. Other patients studied have shown that in cases of chronic parathyroid insufficiency where the use of parathormone must be continued for a long time and probably indefinitely the size of the dose should probably not exceed 5 or 10 units daily.

The Action of Soap on the Animal Organism. By Irvine H. PAge, M.D., (by invitation), and E. V. AlleN, Rochester, Minn. 
A detailed study was made of the effect of injection of soap on white rats, white mice, and rabbits. It was found that soap injected intravenously or intraperitoneally produced extremely toxic conditions and a variety of pathologic changes in the tissues. Chemical analysis of the livers and kidneys showed that in general the total fat was decreased and the iodine number increased. Pharmacologic studies disclosed that the soap lowered blood pressure, stimulated respiration, produced cardiac irregularities and increased the permeability of the meninges to dye. The physiologic significance of these experimental observations were considered.

Electrocardiographic Studies on Changes in the Position of the Heart. By M. H. Nathanson, M.D., Minneapolis, Minn.

It has been noted by several observers that the form of the electrocardiogram is altered on change in position of the body, as a result of a shift in the position of the heart. It has been suggested that the absence of such a change may be considered as evidence of adhesive pericarditis, a condition difficult to diagnose. When an individual turns from the reclining position to one side and then the other, there is a rotation of the heart about two axes, (1) antero-posterior, and (2) longitudinal. The electrical effects of these two types of rotation are in the opposite directions and it is theoretically possible that one may neutralize the other, thus affecting no change on the electrocardiogram.

A group of normal individuals and cardiacs in whom a fixed heart was very improbable was studied and a detailed analysis made of the electrocardiographic alterations on change of position and on different phases of respiration. A change in the amplitude of the QRS usually in the first and third leads was practically constant, but a variety of combinations of changes was noted. The most frequent alteration was a change in the direction of right preponderance when the individual was turned on the left side, with a tendency to left preponderance on turning to the right. The effect of such factors as habitus of the individual, size of heart and the presence of pleural adhesions was noted. Observations were also made on three cases of adherent pericardium.

Radical Inclusions of Giant Cells. By EDwIN T. HIRsch, M.D., Chicago, Ill.

Visceral and focal lesions resembling tuberculosis and containing giant cells with radial inclusions are mentioned in fourteen reports now on record. The nature of these inclusions is an enigma, and a wide variety of opinions have been expressed as regards their origin and the significance of the associated lesions. Visceral lesions of this kind in tissues obtained postmortem from the bodies of four patients and focal lesions in twenty other tissues removed surgically are the human tissues that furnish the basis of my report. The results of the chemical analysis of human tissues and those of experiments in animals suggest that the inclusions are modified saturated fats. 
Experimental Production of Arteriosclerosis in the Aorta and Coronary Arteries. By Don C. Sutron, M.D., and M. D. Davis, M.D., (by invitation), Chicago, Ill.

During the past year, in a group of some fifteen or sixteen dogs, the ramus descendens branch of the left coronary artery has been traumatized, the dog sutured and allowed to live. For a period of 200 days or more these dogs have been on a high calcium intake, with doses of parathormone. In this group there have developed arteriosclerotic changes in the aorta, scar tissue around the ramus descendens branch and diffuse scar tissue in the myocardium. Cardiac measurements have been made by $x$-ray and electrocardiographs have been taken at intervals during the course. The final conclusions of all this work will be based on autopsy findings.

Albuminuria in Young Men. By H. S. DieHL, M.D., and C. A. McKInLAY, M.D., Minneapolis, Minn.

The frequency of albuminuria is noted in approximately 20,000 young men who entered the University of Minnesota since 1921. In part of the series, different tests for albumin were used and are compared. On the first examination, 5.24 per cent were found to have albumin; two-thirds of these later had negative reports. Only 6.74 per cent had what seemed to be definite evidence of kidney disease. Under proper control, the group with albuminuria was studied as to age, heightweight percentage, pulse rate, blood pressure, histories of certain past diseases and the condition of the nose and throat. The changes noted, compared to a control group, were a slight reduction in average age and in mean height-weight percentage and a definite increase in the incidence of acute upper respiratory tract infections, of chronic nasal discharge and of abnormal tonsillar conditions.

Auricular Fibrillation as the Only Manifestation of Heart Disease. By W. M.

Fowler, M.D., (by invitation), and Fred M. SMITH, M.D., Iowa City, Iowa.

Ten patients are recorded, in whom the auricular fibrillation was the only evidence of cardiac disease. The oldest was 34 years of age. In one, the irregularity was attributed to stimulation of external auditory canal, one to exertion, four to alcohol, one to carbon monoxide, one to ether anesthesia, and two were attendants in gasoline stations.

In seven, the auricular fibrillation was paroxysmal, while in three, it had persisted from two weeks in two and one-half years. The sinus rhythm was restored in the latter three by quinidine. The patient with the auricular fibrillation of two and one-half years duration later came to necropsy and the heart was regarded normal.

The Effect of Alkali Administration on Gastric Acidity. By L. C. Gatewood, M.D., Chicago, Ill.

In a group of patients undergoing treatment for gastric and duodenal ulcer, an opportunity arose to study the effect of prolonged alkali administration on the 
secretion of hydrochloric acid. The diet was maintained practically constant and the patient was given doses of alkali just sufficient to neutralize the free hydrochloric acid. The quantity of alkali required, furnished, therefore, a measure of the amount of acid secreted. Curves are presented to show the variations in alkali required. In some instances there was a progressive increase in acidity to such high levels that it was not safe to control the acidity in this way. In some the level of acidity remained unchanged over long periods of time. In many the acid secretion decreased progressively to low levels or disappeared for variable periods of time. While the latter change was frequently coincident with the occurrence of alkalosis, it has also occurred with no change in blood chlorides or $\mathrm{CO}_{2}$ combining power of blood.

\section{Tuberculosis Among Nurses. By Everett K. Geer, M.D., Saint Paul, Minn.}

From 1920 to 1928 forty-two of nine hundred and thirty-four nurses (4.5 per cent) at the Ancker Hospital in St. Paul developed tuberculosis. Because of this high incidence an investigation was instituted.

Beginning in September, 1928, all new nurses entering training were given intradermal tuberculin tests along with their customary physical examination and chest $x$-rays. The new group in September, 1928, showed that one-third reacted positively to intradermal tuberculin. The rest of the training school was then tested and only four of one hundred forty-seven were found negative.

Since then all nurses entering training have been tested and if negative re-tested every six months. This study has shown that in the past two years about 30 per cent of nurses entering the Ancker training school are positive and that by the end of their first year nearly all are positive.

The Ancker Hospital has a tuberculosis department of two hundred and fifteen beds and nurses have duty in this department before the end of their first year.

Nurses in other training schools in St. Paul were tested and average 42 per cent reactors in their third year.

Of one hundred and twenty-two new nurses entering the Ancker training school in the past two years seven have developed tuberculous disease. Six of these were negative to $1 \mathrm{mgm}$. of old tuberculin on entering training. Three of the seven have developed pleurisy with effusion, two, parenchymatous lung lesions, one, a basal lesion of the childhood type and one erythema induratum with tracheobronchial adenopathy.

It is felt that by carrying out more rigid contagious technique in the tuberculosis department and by routinely taking a single chest film of all persons admitted to the hospital on all services that the incidence of tuberculous infection and disease among nurses can be materially reduced at Ancker Hospital and these procedures are to be instituted.

It is recommended that especially in those communities where the incidence of tuberculous infection is low among young adults that in tuberculous sanatoria and tuberculous departments of general hospitals, contagious technique should be employed. It is also considered advisable to routinely $x$-ray the chest of all 
admissions to general hospitals to eliminate in part the unrecognized consumptive menace.

The Effect of Amyl Nitrite on Striated Muscle Spasms. By C. D. CHRISTrE, M.D., Cleveland, Ohio.

During the past year it has been found from extensive clinical trials that amyl nitrite when inhaled in sufficient dosage exerts a decided effect on the acute myalgias such as torticollis, lumbago, pleurodynia, etc. In some instances the pain and spasm are entirely relieved; in others the effect, while beneficial, is transient. There are some instances in which clinically the condition would seem to be an acute myalgia, but the inhalation of amyl nitrite gives no relief.

Attempts have been made in the laboratory to produce a condition analagous to acute myalgias, in the muscles of animals, without success. Attempts have also been made to counteract various kinds of experimentally increased muscle tone in animals, from the effect of caffein, nicotine, cardisol, veratum, insulin, tetanus toxin, barium, guanidine, lactic acid, etc., without success, when amyl nitrite was used in a dosage at all comparable with that used in the clinical application of the drug.

Studies in Serum Viscosity in Hypertension. By Edmund F. Foley, M.D., (by invitation), and C. S. WILliamson, M.D., Chicago, Ill.

Blood pressure is essentially the result of the force of the heart beat, the peripheral resistance, and the viscosity of the circulating fluid. It is commonly appreciated that pathological conditions which increase the first two factors are frequently associated with hypertension. This is a study of the third factor or the viscosity in cases of the hypertension of nephritis.

In normal cases the viscosity as determined by Ostwald's method, temperature being controlled to $37^{\circ} \mathrm{C}$. was found to be from 1.794 to 2.07 , average 1.86 . In ten cases of severe nephritis, two of which were in uremia, and all showing marked evidences of renal decompensation, the maximum was 1.464, the minimum 1.351, the average 1.390. The two cases in uremia were less than the average being 1.365 and 1.351. This study would indicate that in nephritis hypertension is not due to increased viscosity of the serum.

A Comparison of the Heat Lost by Vaporization of Water Determined by the Basal Insensible Loss of Weight, with the Basal Heat Production of the Albino Rat. By James A. Greene, M.D., and P. R. LuCE, M.D., (by invitation), Indianapolis, Ind. Introduced by H. W. Gauchat, Canton, Ohio.

The gaseous metabolism was determined by a method similar to Prince's. The insensible loss of weight was obtained on a balance with a sensitivity of $0.5 \mathrm{mgm}$. for a $\mathbf{3 0 0}$ gram load.

The basal heat production compared favorably with that observed by others, and the percentage of heat lost by vaporization of water ranged between 20.63 and 26.80 , with an average of 23.81. No difference was observed in the use of thin, 
obese, and normal animals. The respiratory quotient was found to average 0.72 , under standard conditions, and the insensible loss of weight was composed of 100 per cent of water with this respiratory quotient. Standard conditions were eighteen to twenty-four hours without food or water at temperatures from 25 to $31^{\circ} \mathrm{C}$.

A Study of the Effect of Insulin on Gastric Motility. By T. E. HeInz (by invitation), and W. L. PaLmer, M.D., Chicago, Ill.

It is well known that insulin frequently produces an intense desire for food. Bulatao and Carlson have shown experimentally that it stimulates gastric hunger contractions. Hence it has been generaliy assumed that the hunger observed clinically after insulin administration is due to increased gastric motilty.

In our experiments this has been tested and kymographic records of the gastric activity made. Definite insulin reactions were produced accompanied by a marked lowering of the blood sugar and frequently by a marked increase in the general desire for food.

The relationship between "hunger," "desire for food" and "hunger contractions" is discussed.

Intestinal Hypersensitivity as an Etiological Factor in Angio-neurotic Edema. By Stanley E. Dorst, M.D., Cincinnati, Ohio.

Two patients with histories of well established angio-neurotic edema have been studied with the hope of establishing the existence of an allergic state. Foods, pollens, and the usual stock bacterial extracts were all employed with negative results. Various possible foci were then investigated, including the teeth, sinuses nose, throat, and the enteric tract. All results were insignificant except those obtained using organisms recovered from the enteric tract. Marked reactions followed the intradermal injection of certain colonic strains. Typical attacks could be precipitated within a few minutes by a moderately large dose of the antigen. The patients were desensitized with these antigens with the results that one has gone seven months without an attack and the other fourteen months without symptoms.

Unusual Phagocytosis by Reticular Cells in a Patient Having a Gram-Negative Bacillus in the Blood. By FranK J. HECK, M.D., (by invitation), and H. MILton ConNer, M.D., Rochester, Minn.

The patient, a male, farmer, aged forty-five years, gave a history of ten months of fever, weakness, anemia, increased sense of warmth, sweating, and headache, and had ecchymotic areas on face for two years, with slight dyspnea on exertion. Examination showed a palpable spleen, skin ecchymoses, enlarged liver, positive tourniquet test and varying degrees of fever. Leukocyte count from ear varied between 35,000 and 78,000 . In blood from finger and vein the count was never above $(3,500$.) Morphologically there was unusual phagocytic activity by reticular 
cells, with inclusion of whole polymorphonuclear neutrophiles, neutrophile remnants or erythrocytes. These reticular cells were seen only in blood from the ear and never in blood from the finger or vein. Six out of eight blood cultures on prolonged culture, two weeks or more, showed the presence of a hemophilic small Gram-negative bacillus of the influenza group.

\section{Treatment of Septic Meningitis by Intra-carotid and Cisterna Magna Injections.} By James A. Evans, M.D., LaCrosse, Wis.

Review of literature and discussion of rationale of intra-carotid and intra-cisternal injections of pneumococcus anti-sera and pneumoquin-base for pneumococcus meningitis and anti-meningococcus serum for meningococcus meningitis.

Two cases of pneumococcus meningitis reported treated unsuccessfully by this method. Report of a very fulminating case of meningococcus meningitis treated unsuccessfully by this method.

Lactic Acid in Human Stools. By J. W. Pittman, (by invitation), and W. H. Olmsted, M.D., St. Louis, Mo.

The products of the action of $B$. coli on glucose in culture media are lactic, acetic and formic acids, alcohol, carbon dioxide and hydrogen. In previous work, the amounts of acetic acid found in stools under various conditions were determined and from this data the amounts of carbohydrate metabolized by bacteria were calculated. Since there are two molecules of lactic acid formed for one of acetic. a corollary of our work would be the determination of the amounts of lactic acid found in stools.

Method. The stools were mixed with a solution of 30 per cent mercuric sulphate dissolved in 10 per cent sulphuric acid. The mercury was precipitated by 10 per cent solution of sodium hydrate. After filtering, the traces of mercury were thrown out by hydrogen sulphide. To rid the filtrate of sugar, copper sulphate was added and precipitated with solid calcium hydrate. The final filtrate was water clear. Lactic acid in the filtrate was determined by the method of Friedeman Cotonio and Shaffer.

Results. 1. In human adult stools there are at the most only traces of lactic acid.

2. Added lactic acid to stools may be recovered to the extent of 80 to 85 per cent. Recovery from watery solutions of lactic acid are 92 to 95 per cent.

3. If lactic acid be added to a stool and half of the stool sterilized and the other half incubated, lactic acid disappears from the incubated portion but can be recovered from the unincubated portion.

Conclusions. 1. A rapid method for determination of lactic acid in stools is presented.

2. The bacterial flora of the intestinal tract in the adult individual rapidly destroys lactic acid. 
The Effect of the Combined Use of Ephedrine Hydrochloride and Digitalis or Ephedrine Hydrochloride and Ouabaine on the Unanesthetized Dog. By CARL A. JoHNson, M.D., (by invitation), and N. C. GILBERT, M.D., Chicago, Ill.

By experiments upon unanesthetized dogs and by clinical observations the authors are of the opinion that the effect of the combined use of ephedrine and digitalis bodies in sub-lethal doses is distinctly toxic, especially in cases with small cardiac reserve.

The authors interpret their results as indicating that they are dealing with two cardiac poisons acting upon irritability and conductivity of cardiac tissue. They also feel that ephedrine in the doses given may produce some of the untoward effects observed from a secondary paralysis of the myoneural junction and the smooth musculature of the blood vessel as shown by Koppani and Luckhardt. This may increase the tendency toward a sudden fall in blood pressure.

In conclusion, they emphasize that when digitalis and ephedrine are used on the same patient, they should be given with extreme caution.

\section{The Occurrence of Digitalis in Ascitic and Edema Fluids of Patients Under Treatment} with Digitalis. By George H. MrLler, M.D., Iowa City, Iowa.

Using the Hatcher, cat-assay type of experiment, the ascitic and subcutaneous fluids of certain patients under treatment for cardiac decompensation are shown to contain a digitalis-like substance. The amount of the drug present is sufficient to be of clinical significance if it were to enter the blood stream.

Hatcher has shown that digitalis is not eliminated to any great extent by the kidney. Since patients under effective treatment for cardiac decompensation will often put out enormous amounts of dropsical fluid by way of the kidneys, the possibility of digitalis poisoning from retention and concentration of digitalis must be considered.

We have seen clinical evidence suggestive of such a result when very rapid elimination of fluid was taking place.

Spontaneous Peptic Ulcers of the Duodenum Following the Total Loss of Pancreatic Juice. By Robert Elman, M.D., St. Louis, Mo.

Peptic ulcers were found uniformly in dogs losing the total external secretion of the pancreas for two weeks or longer. The ulcers always developed just distal to the pylorus. The animals were all kept in good general condition and blood normal by intraperitoneal injections of Ringer's solution. Symptoms of gastric irritability were present in all cases. These observations fit in with a number of experiments by other workers and have a significant bearing on the problem of the pathogenesis and treatment of duodenal ulcer in man.

A Note on the Prevention of Spinal Cord Degeneration in Pernicious Anemia. By Paul StarR, M.D., Chicago, Ill.

A survey of the clinical course of eight of our patients having pernicious anemia and spinal cord degeneration indicates that they may be divided sharply into a 
group of four in whom the neurologic symptoms have not progressed and a like number that have grown steadily worse. The first group has kept a count above five million throughout the period of three years' observation while those who have had increasing spinal cord difficulty have had more or less anemia during this period. There is a striking contrast between the average blood counts of these two groups. The anemic patients are not to be classed as liver failures since adequate liver has finally raised the blood count in each case. Neglect or gross infection produced the anemia.

Conclusion. Those patients who received sufficient specific substance to keep the blood count above five million did not have progress of cord symptoms while those who were allowed to become anemic had progressive central nervous degeneration

Segmental Cutaneous Hyperalgesia as an Accurate Indicator of Visceral Disease. By LeE D. CADy, M.D., St. Louis, Mo.

The utilization of the so-called Head zones of visceral pain reference has been almost totally neglected as a diagnostic test for visceral disease. Visceral organs that are inflamed or otherwise under tension usually cause characteristic patterns of hyperalgesia of the skin that are often of great value in differential diagnosis. The author's experience includes a study of these patterns in diseases of the aorta, heart, esophagus, gallbladder, liver, kidney, small intestine, appendix, colon, urinary vesical, prostate, salpinx and uterus.

The Clinical Significance of the Electrocardiogram in Hypersensitive Heart Disease. By Thomas Ziskin, M.D., (by invitation). Introduced by Hugo Altnow, Minneapolis, Minn.

Most observers believe that hypertension is an hereditary disease beginning in middle life, while some believe that it has its beginning in childhood and goes through various clinical stages. A comparative study was made of the clinical, $\mathrm{x}$-ray and electrocardiographic findings in a series of 324 cases of hypertension.

It was found that the average period of onset of hypertension was in the fourth decade and that the condition runs a progressive course, reaching its maximum during the sixth decade. The size of the heart showed a corresponding progressive increase.

By means of the electrocardiogram, we can divide hypertensive heart disease into three stages: the irritative stage, the quiescent stage and the degenerative stage. The absence of the isoelectric phase in the S-T interval was characteristic of the irritative stage. The presence of the inversion of the $T$ wave, arborization block and delayed conduction was characteristic of the degenerative stage. There was no definite relationship between the height of the blood pressure, the size of the heart and left ventricular preponderance. The presence of a depression or elevation of the S-T phase and other signs of myocardial degeneration was noted more frequently as the blood pressure increased. 
The earlier in life a systolic blood pressure of 190 was reached, the more rapid was the course of the disease and the greater the damage to the heart muscle.

Serial Electrocardiographic Studies in Acute Coronary Thrombosis. By Hugo A. Freund, M.D., and WARRen B. Cooksey, M.D., (by invitation), Detroit, Mich.

In the past two and one-half years nineteen cases of coronary thrombosis were seen in private practice. By means of the portable electrocardiograph frequent tracings were made and in every one of the nineteen cases, evidence which we feel may be said to be diagnostic of coronary thrombosis was obtained. These findings are: (1) Changes from the isoelectric level, of the S-T interval; or (2) development of the coronary $T$ wave first described by Pardee; or (3) flattening or other changes in the $T$ wave occurring in a short period of time; or (4) marked sudden fall in voltage of the $Q$. R. S. In six cases electrocardiographic tracings had been obtained previous to the infarction. Nine cases are dead and either the type of death or autopsy has verified the diagnosis. Lantern slides are to be shown summarizing the electrocardiographic changes in these cases, and a colored plate of a typical thrombosis with infarction.

Blood Urobilin in Nitrogen Retention. By M. A. Blankenhorn, M.D., Cleveland, Ohio.

Thirty patients with high blood urea were tested by estimating the blood urobilin according to a method previously described by the author. The average is compared with the average blood urea and uric acid. It appears that blood urobilin is "retained" when there is retention of nitrogen and to approximately the same degree.

Thyrotoxicosis with a Normal Metabolism Early in the Course of the Disease. By

Fred E. Ball, M.D., Chicago, Ill.

Occasionally patients are seen who present symptoms and physical findings of a thyrotoxicosis but who have normal metabolic rates. Three such cases are reported who later developed an increased metabolic rate within a period of a few weeks to two or three months. The symptoms did not change during that time. The suggestion is made that possibly some cases may be diagnosed as nervous when in reality they have a thyrotoxicosis.

Hypometabolism in Youth and Young Adult Life. By JoHN TUCKer, M.D., and E. P. McCullaGH, M.D., (by invitation), Cleveland, Ohio.

1. A study of hypometabolism in individuals between the ages of $\mathbf{1 5}$ and $\mathbf{3 0}$ years (inclusive).

2. Comparative study of two groups of these cases:

(a) Those in which there is a slight lowering of the metabolic rate.

(b) Those in which there is a marked lowering of the metabolic rate. 
3. Comparative study of these two groups of cases with relation to prominent symptoms:

(a) Those in which prominent symptoms of neurocirculatory asthenia are noted.

(b) Those in which the symptoms are more indicative of true hypothyroidism.

4. A comparative study of the two groups of cases described above with regard to (1) symptoms, (b) physical signs, and (c) laboratory findings.

5. A study of the response in cases in these groups to the administration of thyroid extract.

Hepatogenous Infection of Gallbladder. By Edmund ANDRews, M.D., Chicago, Ill.

The rich bacterial flora of the normal liver, especially the presence of Welch bacilli. The increase in this flora and its spread to adjacent organs under experimental toxic conditions in the peritoneum. The sterility of normal dogs' bile. The prompt invasion of the gallbladder with microorganisms from the liver in cases of experimental cystic or common duct ligation. Evidences that this infection is hepatogeneous because of the fact that the inflammatory reaction in the gallbladder is much more extensive on its hepatic surface and in early cases of cholecystitis is confined to the hepatic surface of the gallbladder. 